\title{
Stabbing nails into the neck: an unusual self-damaging behavior mandating neurosurgery
}

Patients with Munchausen's syndrome deliberately simulate symptoms of an illness to gain hospital admission and the attention of healthcare providers. Although rare, it is possibly under- recognized and leads to diagnostic dilemmas. ${ }^{1}$ The main features of the condition include the recurrent nature of the illness, repeated similar presentations, visiting different hospitals with the same complaint, and leaving treatment once the motive is discovered. The usual presentations are neurological or abdominal complaints. ${ }^{2}$ The origin of the condition is unknown but there is a suggestion that patients may have sustained brain damage prior to the beginning of their hospital addiction. Since their motivation is unknown, personality disorder and psychopathy have been suggested as probable causes. ${ }^{3}$

Neurosurgery related cases of Munchausen's syndrome generally involve chronic back pain ${ }^{4}$, postsurgery Munchausen's syndrome ${ }^{5}$, or head trauma and intracranial hemorrhage. ${ }^{6}$ These patients spend most of their lives in hospitals with dramatic symptoms and submit to many unnecessary operations and medical investigations.

We report the case of a 38 year-old woman referred with the chief complaint of severe neck pain. Publication of the case material was approved by the ethics committee of Firouzgar hospital in affiliation with Tehran University of Medical Sciences

On history, she mentioned a recent car accident (4 months earlier) leading to minor loss of consciousness without amnesia. Brain CT scan, at that time, was normal and she recovered completely. There was no history of psychiatric disorder and she lived, problem-free, with her husband of 20 years and two sons. Her mother had died when she was seven years old and she grew up with her mother-in-law. She gave no history of physical or emotional abuse in her childhood. X-ray of her neck detected a needle in the soft tissue posterior to the second vertebra. There was no sign of skin injury demonstrating the entrance of the needle. Also, she did not mention any incidents accounting for the needle in this area of her body and denied self-inflicted injury. The needle was removed surgically, and the patient was discharged two days later in good condition. One month

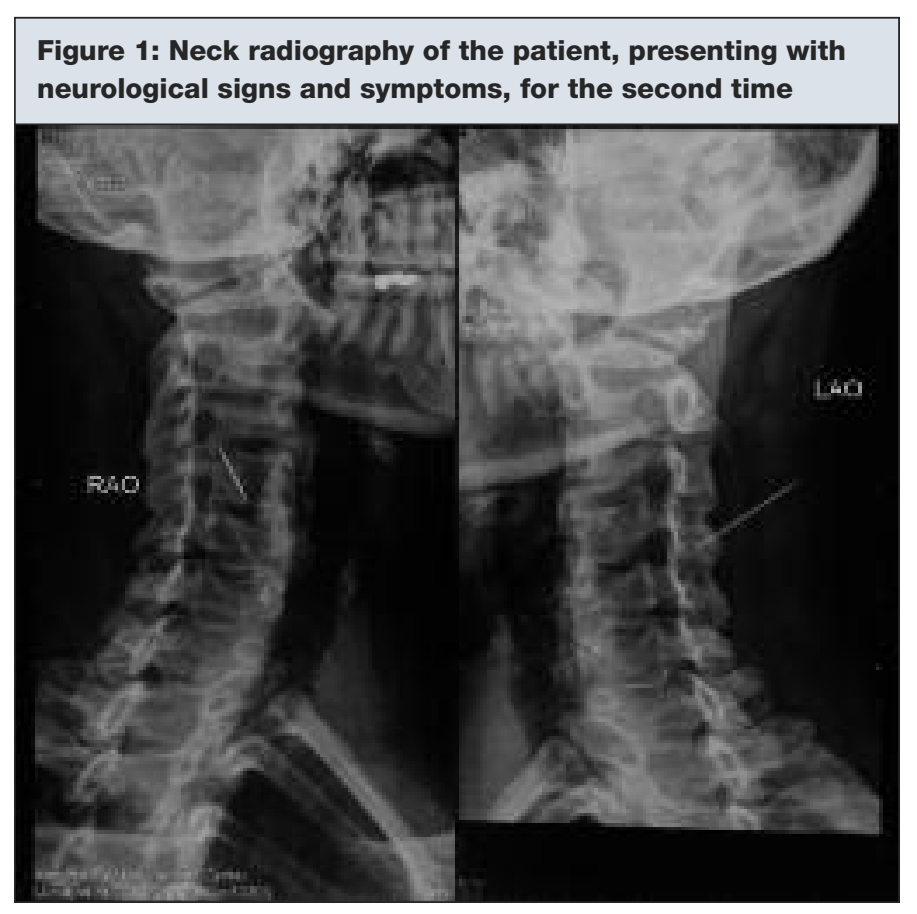

later, she returned with right upper limb weakness and pain in the neck. At imaging, a new nail was detected in her neck (Figure 1) mandating further surgery for its removal.

Factitious disorders, including Munchausen's syndrome, are likely to be encountered by all clinicians during their career. ${ }^{7}$ From the view of neurosurgeons, Munchausen's syndrome is a rare psychiatric diagnosis, especially in postoperative patients. The common clues for diagnosis of such condition are non-healing wounds in the absence of risk factors for wound failure, a positive history of psychiatric illness, repeated bleeding from the wound site, and a desire to continue in the sick role. Early detection of this condition allows appropriate psychiatric referral thus preventing iatrogenic injuries, abuse of the medical system, unnecessary operations, and further injuries that may be harmful. 5 Typically, patients present as acute emergency cases with a plausible medical and social history later found to be entirely fabricated. In spite of the physicians' best efforts, and possible surgery, no diagnosis can be made. The patient then discharges him/herself and goes to another hospital to repeat the process.

The condition is most often seen in adolescent females with disturbed body image and interpersonal difficulties. 
Deprivation and physical or sexual abuse, feelings of internal emptiness, and unbearable psychic tension are potential psychodynamic causes of this act.

Psychotherapeutic treatment strategies focus on enhancing ability to express emotions, caring for the patient's own body, and establishing confidential and stable relationships. ${ }^{8}$ Physicians should keep this diagnosis in mind when confronting a patient with pathology that cannot be explained by the patient's history, as demonstrated by the above case. Although this patient had no previous psychological problems, she performed potentially life-threatening self-harm behavior on two occasions, the second of which was accompanied by serious neurological complications. The potential for suicide is also present.

Consultation with a psychiatrist can help the primary treatment team manage the patient in the safest and most appropriate way, for instance by avoiding unnecessary procedures, preventing further self-harm, and avoiding angry or threatening interactions with the patient. This approach, as well as a flexible and creative treatment plan, emphasizing the consistency of care, will certainly improve the patient's prognosis. ${ }^{9}$ Forensic medicine specialists may see these patients in the emergency room of the hospitals. They, too, should be aware of this psychiatric condition and help the treating staff to manage the patient and prevent possible legal consequences. At post-mortem, in cases with a history of psychiatric problems, such bizarre self-damaging behavior should be borne in mind.
A Aghabiklooei ${ }^{1}$, R Hashemi ${ }^{2}$, N Zamani ${ }^{3}$ 1Deaprtment of Forensic Medicine and Clinical Toxioclogy, Firouzgar Hospital, Tehran University of Medical Sciences,

Tehran, Iran;

2Department of Neurosurgery, Firouzgar hospital, Tehran University of Medical Sciences, Tehran, Iran; 3Department of Clinical Toxicology, Loghman Hakim Hospital, Shahid Beheshti University of Medical Sciences, Tehran, Iran

\section{References}

1. Doherty AM, Sheehan JD. Munchausen's syndrome--more common than we realize? Ir Med J 2010;103:179-81.

2. Hariharasubramony A, Chankramath S, Srinivasa S. Munchausen syndrome as dermatitis simulata. Indian J Psychol Med 2012;34:94-6.

3. Clarke E, Melnick SC. The Munchausen syndrome or the problem of hospital hoboes. Am J Med 1958;25:6-12.

4. Callegari C, Bortolaso P, Vender S. A single case report of recurrent surgery for chronic back pain and its implications concerning a diagnosis of Münchausen syndrome. Funct Neurol 2006;21:103-8.

5. Lad SP, Jobe KW, Polley J, Byrne RW. Munchausen's syndrome in neurosurgery: report of two cases and review of the literature. Neurosurgery 2004;55:1436.

6. Banerjee AK. Trauma and Munchausen's syndrome. Arch Emerg Med 1991;8:217-8.

7. Folks DG. Munchausen's syndrome and other factitious disorders. Neurol Clin 1995;13:267.

8. Hönigl D, Kriechbaum N, Zidek D, Hasiba K, Zapotoczky HG. [Selfinjury behavior]. Acta Med Austriaca 1997;24:19-22.

9. Huffman JC, Stern TA. The diagnosis and treatment of Munchausen's syndrome. Gen Hosp Psychiatry 2003;25:358-63. 